\title{
Wilson's disease: Update on pathogenesis, biomarkers and treatments
}

Samuel Shribman ${ }^{1}$, Aurelia Poujois ${ }^{2}$, Oliver Bandmann ${ }^{3}$, Anna Czlonkowska ${ }^{4}$ and Thomas T Warner ${ }^{1}$

1. Reta Lila Weston Institute, UCL Queen Square Institute of Neurology, London, UK

2. National Reference Centre for Wilson's Disease, Department of Neurology, Rothschild Foundation Hospital, Paris, France.

3. Sheffield Institute of Translational Neuroscience, Sheffield, UK

4. Second Department of Neurology, Institute of Psychiatry and Neurology, Warsaw, Poland.

\section{Corresponding author}

Dr Samuel Shribman

Reta Lila Weston Institute

UCL Queen Square Institute of Neurology

1 Wakefield Street, London, WC1N 1PJ

s.shribman@ucl.ac.uk

\section{Short title}

Update on Wilson's disease

\section{Word count}

Abstract: 233

Manuscript: 5,000 


\section{ABSTRACT}

Wilson's disease is an autosomal-recessive disorder of copper metabolism caused by mutations in $A T P 7 B$ and associated with neurological, psychiatric, ophthalmological and hepatic manifestations. De-coppering treatments are used to prevent disease progression and reduce symptoms, but neurological outcomes remain mixed. In this article, we review the current understanding of pathogenesis, biomarkers and treatments for Wilson's disease from the neurological perspective, with a focus on recent advances. The genetic and molecular mechanisms associated with ATP7B dysfunction have been well characterised but, despite extensive efforts to identify genotype-phenotype correlations, the reason why only some patients develop neurological or psychiatric features remains unclear. We discuss pathological processes through which copper accumulation leads to neurodegeneration, such as mitochondrial dysfunction, the role of brain iron metabolism and the broader concept of selective neuronal vulnerability in Wilson's disease. Delayed diagnoses continue to be a major problem for patients with neurological presentations. We highlight limitations in our current approach to making a diagnosis and novel diagnostic biomarkers, including the potential for newborn screening programmes. We describe recent progress in developing imaging and wet (fluid) biomarkers for neurological involvement, including findings from quantitative MRI and other neuroimaging studies, and the development of a semi-quantitative scoring system for assessing radiological severity. Finally, we describe the use of established and novel chelating agents, paradoxical neurological worsening, and progress developing targeted molecular and gene therapy for Wilson's disease, before discussing future directions for translational research. 


\section{INTRODUCTION}

Wilson's disease is an autosomal recessive disorder of copper metabolism caused by $A T P 7 B$ mutations. Originally described as hepatolenticular degeneration, it classically presents with the combination of liver disease and a movement disorder during adolescence or early adulthood, albeit with a highly variable phenotype. Up to $60 \%$ of patients have neurological or psychiatric symptoms at onset, and are referred to as having neurological presentations, irrespective of the severity of any underlying liver disease. ${ }^{1}$ Patients presenting primarily with liver disease ranging from asymptomatic deranged liver function tests, hepatomegaly or splenomegaly to acute liver failure are referred to as having hepatic presentations. Chelating agents are used to 'de-copper' patients but neurological outcomes are unpredictable; symptoms usually improve however, a minority have persistent or progressive neurological disability. ${ }^{2,3}$

Our understanding of genetic and molecular mechanisms, neuroimaging abnormalities and other biomarkers for neurological involvement in Wilson's disease has advanced in recent years. Novel chelating agents, adeno-associated virus-based gene therapy and variant-specific treatments are also in development and may help improve neurological outcomes. Clinical presentations and hepatic aspects of Wilson's disease have been comprehensively covered elsewhere. In this narrative review, we discuss the pathogenesis, biomarkers and treatment of Wilson's disease from the neurological perspective, with a focus on recent developments and future directions. 


\section{PATHOGENESIS}

\section{Structure and function of ATP7B}

ATP7B is a copper-transporting P-type ATPase that consists of six metal-binding domains and eight transmembrane domains, which form a pore for ATP-dependent transport of copper across membranes. ${ }^{4}$ This protein has two specific functions in hepatocytes. Firstly, it supplies copper to the trans-Golgi network (TGN) for incorporation into caeruloplasmin, the main copper-transport protein that is secreted into the bloodstream. Secondly, it facilitates the biliary excretion of excess copper by translocating to late endosomal or lysosomal compartments and sequestering copper into vesicles for export across the apical (canalicular) membrane.

ATP7B is expressed in other human tissues. Davies et al found immunohistochemical staining for ATP7B in all human brain regions they tested including the striatum, substantia nigra, anterior cingulate and visual cortices and cerebellum. ${ }^{5}$ Animal studies suggest that ATP7B indirectly regulates the activity of copper dependent enzymes, such as dopamine beta-hydroxylase and copper/zinc superoxide dismutase-1 (SOD1), in specific neuronal subpopulations. ${ }^{6}$ It also interacts with ATP7A, another copper-transporting ATPase that is widely expressed in the brain. ${ }^{7}$ Interestingly, alternative splicing of $A T P 7 B$ mRNA in the brain was described more than two decades ago. This suggests different isoforms of ATP7B may be expressed in neurons and glia but has not, to our knowledge, been explored further. ${ }^{8}$

\section{Genetics}

Over 900 pathogenic mutations in $A T P 7 B$ have been reported although ever some variants with high allele frequencies probably exhibit low penetrance. ${ }^{9}$ The majority of mutations are missense, nonsense or frameshift mutations. Additional mechanisms including exon skipping, large deletions and intronic variants have recently been described but are probably rare. The most common mutation among patients from Northern and Eastern Europe is H1069Q but its frequency varies significantly between countries. In a genetic study of 248 patients from Poland, $45 \%$ were homozygous and $41 \%$ were heterozygous for this mutation. ${ }^{10}$ In a similar study of 181 patients from the UK, H1069Q mutations were identified in $19 \%$ of patients. ${ }^{11}$ Other variants are much more prevalent in non-European populations. For example, R778L is the most common mutation among patients from China.

The genetic basis for phenotypic variation remains elusive despite extensive efforts to identify genotype-phenotype correlations. There was no association between $A T P 7 B$ genotype and the presence of neurological involvement in a cohort of 1,172 European patients. ${ }^{12}$ Patients with I1148T or R919G variants were more likely to have neurological involvement in a cohort of 1222 Chinese patients, 
although both variants were rare. ${ }^{13}$ Several genetic modifiers have been proposed. Schiefermeier et al and Litwin et al found an association between the $\varepsilon 3 / \varepsilon 3$ ApoE genotype and delayed onset of symptoms in H1069Q homozygotes, particularly women, but not with clinical presentation. ${ }^{14}$ Roy et al subsequently suggested that $A P O E$ and $P R N P$ genotypes may be associated with cognitive or behavioural symptoms. ${ }^{15} \mathrm{~A}$ whole-exome sequencing study examining genes in the ATP7B interactome suggested rare variants in ESD and INO80 might also affect phenotype. ${ }^{10}$

\section{Molecular mechanisms}

The functional consequences of the most common $A T P 7 B$ variants have now been characterised. The H1069Q and R778L variants cause retention of ATP7B in the endoplasmic reticulum (ER) leading to impaired copper export. Using hepatocyte-like cells derived from patients with homozygous H1069Q mutations, Parisi et al recently demonstrated that around two thirds of the mutant protein is retained in the ER where it undergoes more rapid degradation. However, the ability to recruit ATP7B to the TGN in response to increasing copper is preserved with important implications for using targeted molecular therapies to rescue ATP7B function. ${ }^{16}$

Huster et al explored the effects of a further 27 variants in Sf9 and HEK293 cells. ${ }^{17}$ There were marked differences in copper transport, phosphorylation activity, protein expression and subcellular localisation between variants. In a similar study, Roy et al characterised the functional properties of further variants demonstrating differential effects on intracellular copper concentrations. ${ }^{18}$ Intriguingly, they also found that co-expressing the G1061E variant, which is usually retained in the ER, with the A595T variant restored localisation of ATP7B to the TGN. ATP7B has previously been shown to form stable dimers in vitro and so genetic and functional studies may need to consider interactions between heterozygous variants in the future.

\section{Neurodegeneration}

An inability to excrete copper in bile leads it to accumulate in the liver with subsequent accumulation of copper in other organs, including the brain. Post-mortem studies confirm that patients with Wilson's disease have dramatically increased copper content in the basal ganglia and cortex. ${ }^{7,} 19$ However, advanced liver disease is not necessarily a prerequisite to developing neurological disease. Przybylkowski et al characterised the liver disease in newly-diagnosed patients with neurological patients and found that, while every patient had at least one feature of liver disease on ultrasound examination, around half of patients had clinical or radiological features of cirrhosis. ${ }^{20}$ In a cohort of 
131 patients who underwent liver biopsy at diagnosis, Ferenci et al found that 8 of 34 patients with neurological presentations had normal histology or steatosis (without hepatitis fibrosis or cirrhosis). Interestingly, four patients with neurological presentations (and two pathogenic ATP7B mutations) had hepatic copper content less than $250 \mu \mathrm{g} / \mathrm{g}$ (dry weight), including one patient with hepatic copper content $50 \mu \mathrm{g} / \mathrm{g} .{ }^{21}$ Low hepatic copper content may result from sampling error, where an area of relatively normal tissue is taken during liver biopsy, however the possibility of neurological involvement developing without increased liver copper content cannot be excluded. Patients may also have increased brain copper content without developing neurological or psychiatric symptoms. In a cohort of 11 patients who underwent brain copper quantification post-mortem, all had dramatically increased copper content in the putamen and frontal cortex, irrespective of presentation. ${ }^{22}$ It therefore appears that a model where copper accumulates in the liver, overflows into the systemic circulation and is then deposited in the brain may be an oversimplification, and that a subset of patients seem to be more vulnerable to developing neurodegeneration in response to copper deposition in the brain, although the mechanism for this is unclear.

Histopathological changes in the brain are heterogeneous and include neuronal loss, spongiosis, cavitation, demyelination and reactive astrogliosis. ${ }^{7}$ These findings can be seen in the basal ganglia, thalamus, brainstem and cerebral white matter but are usually most prominent in the putamen. Animal models of Wilson's disease do not produce a convincing neurological phenotype but do accumulate copper in the brain and have provided some insights on the mechanisms by which this could lead to neuropathology. ${ }^{23}$ Mitochondrial dysfunction appears to play a critical role and brain iron metabolism is also affected. These processes and the broader concept of selective neuronal vulnerability in Wilson's disease are discussed below:

\section{Mitochondrial dysfunction}

Copper is an essential cofactor for energy production in mitochondria but has deleterious effects on the organelle in excess. The prevailing view up until recently has been that copper-induced mitochondrial dysfunction is mediated by the generation of reactive oxygen and nitrogen species in Fenton-like reactions. However, several findings point towards alternative mechanisms for mitochondrial dysfunction:

- Marked mitochondrial copper accumulation, with concentrations up to 200 times normal, occurs in the hepatocytes from $A T P 7 B$ knockout rats prior to signs of oxidative damage. ${ }^{24}$ 
- Loss of mitochondrial membrane potential and reduced ATP production develop in response to exogenous copper in healthy rat brain prior to the increased generation of reactive oxygen species, potentially as a result of direct interactions with protein thiol groups. ${ }^{25}$

- Copper interrupts mitochondrial DNA replication leading to mitochondrial DNA depletion in human blood samples independently of oxidative stress-mediated damage. ${ }^{26}$

These observations suggest that copper toxicity causes mitochondrial dysfunction through both direct and indirect mechanisms. However, studies using induced pluripotent stems cell (iPSC) models of human neurons and glia are needed to further elucidate the precise mechanisms linking mitochondrial dysfunction to neuronal and glial cell death.

\section{Iron metabolism}

Several converging lines of evidence indicate brain iron metabolism is disrupted in Wilson's disease. Susceptibility-weighted imaging (SWI) show increased signal in the basal ganglia of patients with neurological involvement. ${ }^{27}$ Cerebral iron uptake, measured using ${ }^{52} \mathrm{Fe}$-citrate PET imaging, is also increased. In a histopathological study of nine patients, Dusek et al confirmed that SWI abnormalities in the caudate, putamen and pons represent abnormal iron deposition, mainly caused by an increase in iron-containing macrophages, and identified an association between iron accumulation and pathological severity in the putamen. ${ }^{28}$ The basis for abnormal iron deposition in Wilson's disease is unclear however copper-dependent enzymes play an important role in iron metabolism. For example, caeruloplasmin, which is also expressed in the brain, exhibits ferroxidase activity and loss of function in acaeruloplasminaemia leads to brain iron accumulation. ${ }^{28}$

\section{Selective neuronal vulnerability}

There are several potential reasons why neurons, specifically those within the basal ganglia, might be particularly vulnerable to copper toxicity in Wilson's disease:

- Medium spiny neurons of the striatum and dopaminergic neurons of the substantia nigra have unusually high energy requirements making them more susceptible to perturbations in mitochondrial function. ${ }^{29}$

- Endogenous copper-scavenging proteins, metallothionein 1/2 (MT1/2) and Cu/Zn superoxide dismutase (SOD1), are differentially expressed between neuronal subpopulations and glia. ${ }^{25}$

- Copper can directly modulate neurotransmission in the basal ganglia; it reduces tonic inhibition mediated by extra-synaptic GABA receptors in striatal neurons. ${ }^{30}$ 


\section{BIOMARKERS}

\section{Diagnostic biomarkers}

Diagnostic delays are common and represent a missed opportunity to start chelation therapy and prevent neurological disability. In a cohort of 163 patients from Germany, neurological presentations were associated with a significantly longer time from onset of symptoms to diagnosis than hepatic presentations (44 v 14 months). ${ }^{3}$ The mean interval in patients diagnosed in Ireland between 19902011 was 21 months and had not improved over the preceding four decades. ${ }^{31}$ The longest delays are often in patients who initially present with psychiatric symptoms. ${ }^{32}$

Delays usually arise because the clinical features associated with neurological presentation, which are outlined in Table 1 , are highly variable and initially misattributed to more prevalent disorders. In our experience, some patients are incidentally found to have deranged liver function tests or an isolated thrombocytopenia indicative of cirrhosis or hepatomegaly in the years before presenting with neurological or psychiatric symptoms. However, the lack of a simple diagnostic test that can confirm or exclude Wilson's disease compounds the issue. Here, we discuss the current approach to making a diagnosis and two novel diagnostic biomarkers.

\section{Current approach}

A combination of tests is usually required to make the diagnosis. A working group at an international meeting on Wilson's disease in 2001 proposed a diagnostic scoring system that integrates clinical features with serum caeruloplasmin, 24-hour urinary copper, slit lamp examination (for KayserFleischer rings), ATP7B genotyping and hepatic copper content (on liver biopsy) to determine whether Wilson's disease is highly likely, probable or unlikely. ${ }^{33}$ However, in our experience, most neurologists and hepatologists initially arrange a full blood count, liver function tests and serum caeruloplasmin and investigate more thoroughly with a 24-hour urine collection and slit lamp examination when the serum caeruloplasmin is low, neuroimaging is abnormal or they already suspect Wilson's disease.

Neurologists should however be aware of the pitfalls of this approach. While a very low caeruloplasmin $(<0.1 \mathrm{~g} / \mathrm{L})$ is highly suggestive of Wilson's disease, intermediate concentrations $(0.1-$ $0.2 \mathrm{~g} / \mathrm{L}$ ) are less specific and up to $15 \%$ of patients with neurological presentations have normal concentrations $(>0.2 \mathrm{~g} / \mathrm{L}) .{ }^{34}$ There are also methodological concerns about the widely used immunological assay for caeruloplasmin, which also detects apo-caeruloplasmin and may overestimate results. We therefore advocate a low threshold for arranging a urine collection and slit lamp examination with a serum caeruloplasmin at initial assessment, particularly in patients with 
suspected liver disease or a positive family, and promptly arranging further investigations in patients found to have a serum caeruloplasmin less than $0.2 \mathrm{~g} / \mathrm{L}$ or specific neuroimaging abnormalities, discussed further below.

The urinary copper output is increased $(>0.64 \mu \mathrm{mol} / 24$ hours) and Kayser-Fleischer (KF) rings are present in $78 \%$ and $90 \%$ of neurological presentations, respectively. ${ }^{34}$ The diagnosis of Wilson's disease can therefore be established using a combination of serum caeruloplasmin, 24-hour urinary copper and slit lamp examination in the vast majority of patients with neurological presentations. $A T P 7 B$ sequencing, which may take several months, may be required to confirm the diagnosis in a minority of cases. Liver biopsy is very rarely needed for diagnostic purposes in patients with neurological presentations. ${ }^{35}$ Copper incorporation tests that involve administering a radioactive ${ }^{64} \mathrm{Cu}$ isotope (intravenously) or non-radioactive ${ }^{65} \mathrm{Cu}$ isotope (orally) and indirectly measuring incorporation of exogenous copper into caeruloplasmin over 48-72 hours may be helpful if other tests are inconclusive but, to our knowledge, are only available in a few countries. ${ }^{36,37}$

\section{Novel diagnostic biomarkers}

An alternative approach to measuring serum copper has been proposed in the last decade. The exchangeable copper reflects the labile fraction of copper bound to albumin and other peptides and is measured by adding ethylenediaminetetraacetic acid (EDTA) to a serum sample and performing ultrafiltration prior to copper quantification. Levels are higher in patients with extra-hepatic involvement ( 2.75 vs $1.26, \mathrm{P}<0.001)$ and correlate with neurological severity $(\mathrm{r}=0.45, \mathrm{P}=0.016)$ semiquantitative scores for $\mathrm{KF}$ rings $(\mathrm{r}=0.46, \mathrm{P}=0.014)$ and neuroradiological involvement $(\mathrm{r}=0.46$, $\mathrm{P}=0.048) .{ }^{38}$ Expressed as percentage of total serum copper, relative exchangeable copper (REC) appears to be a valuable diagnostic test with a high sensitivity and specificity. With a cut-off of $15 \%$, REC discriminates patients with Wilson's disease from heterozygous ATP7B carriers and healthy controls. ${ }^{39}$ While this test can differentiate Wilson's disease from other causes of liver disease in children and adults, data on its ability to differentiate Wilson's disease from other neurological and psychiatric disorders is not yet available.

Collins et al recently proposed that direct measurements of ATP7B peptides on dried blood spots using immunoaffinity-enriched mass spectrometry as a novel diagnostic test for Wilson's disease. ${ }^{40}$ In a retrospective cohort of 264 patients and 150 healthy controls, they report an overall sensitivity of $92 \%$ and specificity of $98 \%$. ATP7B peptide concentrations were below diagnostic cutoff values in 171/184 (93\%) with a low serum caeruloplasmin $(<0.2 \mathrm{~g} / \mathrm{L})$ and $14 / 16(88 \%)$ with a normal serum caeruloplasmin $(>0.2 \mathrm{~g} / \mathrm{L})$. This suggests that a combination of ATP7B peptide quantification and serum caeruloplasmin is likely to be improve diagnostic accuracy. Nonetheless, the 
ability to confirm or exclude Wilson's disease on a dried blood spot has the potential to reshape our approach to investigating Wilson's disease, enabling more widespread testing of patients presenting to primary and secondary care services with neurological or psychiatric symptoms. It also revives the possibility of newborn screening programmes for Wilson's disease after previous attempts, based on

caeruloplasmin, were largely disappointing. ${ }^{41}$ These findings have important implications but need to be validated in larger, prospective cohorts that include disease controls.

\section{Biomarkers for neurological involvement}

The urinary copper output and non-caeruloplasmin-bound ('free') copper, which is calculated from the serum caeruloplasmin and serum copper, are currently used to monitor treatment response with chelation therapy. However, these conventional copper indices do not differ between patients with hepatic and neurological presentations or correlate with clinical neurological severity, measured using the Unified Wilson's Disease Rating Scale (UWDRS). ${ }^{42}$ Significant progress has been made in understanding and developing end-organ biomarkers that directly measure neurological involvement in recent years. These could potentially be used to guide chelation therapy or predict response to treatment and, in a research setting, for cohort stratification or as secondary outcomes measures in clinical trials. Here, we discuss recent advances in neuroimaging and the use of wet and ophthalmological biomarkers for measuring neurological involvement in Wilson's literature.

\section{Neuroimaging}

Hyperintense signal abnormality in the basal ganglia, thalamus and/or brainstem on T2-weighted (or FLAIR) sequences are characteristic of Wilson's disease and seen in $90 \%$ of patients with neurological presentations (Figure 1). ${ }^{43}$ Those in the putamen may be associated with dystonic features whereas those in the thalamus may be associated with tremor or ataxia and in the midbrain with parkinsonism. However, these clinico-radiological correlations have not been systematically tested. The face of the giant panda sign, where hyperintense signal abnormality surrounds the red nucleus and substantia nigra, is highly specific for Wilson's disease but only seen in a minority of patients.

Dusek et al recently proposed a semi-quantitative MRI scale for measuring neuroradiological abnormalities in Wilson's disease that includes an acute toxicity subscore based on the distribution and severity of these hyperintense signal abnormalities. In a validation study that included 39 treatment-naïve patients, the acute toxicity subscore improved with treatment $(\mathrm{P}=0.002)$ but did not correlate with UWDRS scores at baseline or after two years. ${ }^{44}$ A chronic damage subscore, based on brain atrophy and hypointense signal abnormalities on $\mathrm{T} 2 / \mathrm{T} 2 * /$ susceptibility-weighted imaging, correlated with UWDRS scores at baseline $(\mathrm{r}=0.59, \mathrm{P}=0.005)$ and 24 months $(\mathrm{r}=0.68, \mathrm{P}<0.001)$. 
Quantitative MRI analyses have provided further insights on brain atrophy, susceptibilityrelated changes and diffusion abnormalities in Wilson's disease. Relevant studies are listed in Table $\underline{2}$ (with references in supplementary material), some of which were used to inform the aforementioned semi-quantitative MRI scale. Volumetric studies demonstrate that patients with neurological presentations have atrophy and increased susceptibility in regions of interest including the caudate, putamen, pallidum and thalamus at diagnosis. Dusek et al recently confirmed Wilson's disease causes a predominantly central pattern of atrophy using deformation and surface-based morphometry. The overall degree of brain atrophy also correlates with neurological severity. Smolinski et al calculated the volume of deep grey matter $(\mathrm{r}=-0.46, \mathrm{P}=0.001)$, peripheral grey matter $(\mathrm{r}=-0.52, \mathrm{P}<0.001)$ and white matter $(\mathrm{r}=-0.60, \mathrm{P}<0.001)$ in treatment-naïve patients and found that each correlated with UWDRS scores. In addition, they identified an association between brain volume and serum noncaeruloplasmin-bound copper $(\mathrm{r}=-0.39, \mathrm{P}=0.008)$. Diffusion tensor imaging studies demonstrate decreases in fractional anisotropy and increases in mean diffusivity in the white matter of patients with neurological presentations. In a prospective, longitudinal study, Lawrence et al showed that these indices improve over the first two years of treatment.

Importantly, the cognitive and psychiatric aspects of Wilson's disease have received relatively little attention in the quantitative MRI studies. Executive dysfunction associated with Wilson's disease is assumed to be related to basal ganglia dysfunction but evidence to demonstrate this is so far limited. Neuroimaging correlates for psychiatric symptoms, which are a major source of morbidity for patients with Wilson's disease, have not yet been described.

Several other neuroimaging modalities have been used to study neurological involvement in Wilson's disease (Table 3). Magnetic resonance imaging (MRS) demonstrates that N-acetyl-aspartate (NAA) to creatinine $(\mathrm{Cr})$ ratios are consistently reduced in the basal ganglia of patients with neurological involvement and that myo-inositol $(\mathrm{mI})$ is increased in patients with porto-systemic shunting. Single photon emission computed tomography (SPECT) confirm pre- and post-synaptic dopaminergic dysfunction is common in Wilson's disease and correlates with motor function and that cerebral hypoperfusion is seen in the striatum and scattered cortical areas. Transcranial sonography (TCS) studies have shown that hyperechogenecity of the putamen is seen in the vast majority, if not all, patients with neurological presentation. This can accurately differentiate patients with Wilson's disease from healthy controls and patients with early-onset Parkinson's disease and correlates with neurological examination scores but does not improve with treatment. Third ventricular width measured using TCS correlates with UWDRS and Addenbrooke's cognitive examination scores.

Overall, these findings suggest that repeat MRIs are justified to determine whether initial signal changes improve or worsen after initiation of chelation therapy. However, questions remain regarding 
the mismatch between T2-weighted hyperintensities and clinical severity, the ability of measures of brain atrophy to predict clinical outcomes and the role for diffusion and susceptibility-weighted imaging in monitoring response to treatment. Further studies exploring how imaging biomarkers correlate with clinical and biochemical characteristics in the initial stages of treatment and with clinical outcomes in chronically-treated patients are needed.

\section{Other biomarkers}

Neuronal and glial-derived proteins that are promising biomarkers for other neurodegenerative diseases have recently been tested in Wilson's disease. Neurofilament light (NfL), a non-specific marker of neuroaxonal injury that can be measured in plasma samples using single molecule array technology, is higher in patients with neurological than hepatic presentations (8.7 vs 7.0, $\mathrm{P}=0.005)$ and correlates with UWDRS scores in chronically-treated, stable patients $(\beta=0.1, \mathrm{P}=0.003) .{ }^{45}$ Levels of glial-fibrillary acidic protein (GFAP) and tau have also been reported to higher in serum/plasma samples from patients with neurological presentations. ${ }^{46}$ Further studies investigating NfL, GFAP and tau in patients starting treatment for Wilson's disease and age-specific reference ranges are needed however these preliminary findings provide proof-of-concept that non-invasive wet biomarkers could be used to measure neurological involvement in Wilson's disease in the future. ${ }^{45}$

Serial slit lamp examination to confirm disappearance, or exclude recurrence, of KF rings is helpful in some cases. Anterior segment optical coherence tomography (OCT) has recently been proposed as an alternative technique for detecting KF rings and monitoring treatment response that can provide a quantitative measure of copper deposition and may be more sensitive that slit lamp examination. ${ }^{47}$ In a study that included 29 chronically-treated patients with Wilson's disease, 15 had KF rings on anterior segment OCT that were not visible with slit lamp examination highlighting the potential for OCT to supersede slit lamp examination for the detection of KF rings in a clinical setting. Retinal OCT has also been proposed a biomarker for neurodegeneration in Wilson's disease. Thinning of the retinal nerve fibre layer (RNFL) was greater in patients with neurological involvement (88.1 vs 94.8, $\mathrm{P}=0.002)$ and negatively correlated with UWDRS scores in chronically-treated patients $(\beta=-0.95$, $\mathrm{P}=0.008) .{ }^{48}$ The ability of changes in the RNFL or macula to predict outcome in newly-diagnosed patients and associations with neuroimaging abnormalities are so far untested. 


\section{TREATMENT}

\section{First-line treatments}

Chelating agents increase urinary copper excretion and are used to 'de-copper' patients, and then continued lifelong. Zinc salts inhibit intestinal absorption of copper by upregulation of metallothionein and can also be used to treat Wilson's disease. Treatment strategies and dosing regimens vary. Adverse effects, including paradoxical neurological worsening, are relatively common and outcomes are mixed: In a retrospective study of 137 patients presenting with neurological symptoms, $42 \%$ became asymptomatic, $26 \%$ improved but had residual symptoms, $18 \%$ had significant ongoing disability and $8 \%$ died despite seemingly adequate chelation therapy. ${ }^{2}$ In a separate study on long-term outcomes, $20 \%$ of patients with hepatic presentations developed neurological symptoms during the course of their treatment. $^{3}$

\section{Choice of agent}

Penicillamine, Trientine and Zinc are currently the most widely used treatments in Europe and their use is described in detail in the European Association for the Study of the Liver (EASL) clinical practice guidelines on Wilson's disease. Retrospective studies suggest rates of neurological recovery and paradoxical worsening are broadly similar between these three treatments but discontinuation is more likely with Penicillamine. ${ }^{49}$ The relationship between the dose of Penicillamine or Trientine in the initial de-coppering phase of treatment and neurological outcomes is largely unknown but there is a consensus that clinicians should start low and go slow in patients with neurological presentations. Zinc is considered second or third line in some countries given reports that liver disease can progress despite monotherapy.

The role of Tetrathiomolybdate, a chelating agent that can also increase biliary excretion and prevent intestinal absorption of copper, is currently being re-evaluated. Brewer et al compared Ammonium Tetrathiomolybdate to Trientine in 48 patients with neurological presentations also treated with Zinc in a randomised controlled trial in 2006. Worsening was less common in the Tetrathiomolybdate group (4\% vs $26 \%, \mathrm{P}<0.05) .{ }^{50}$ In an open-label phase II trial reported in 2017 , Weiss et al treated 28 patients with Bis-choline Tetrathiomolybdate. At 24 weeks, $71 \%$ achieved the primary end-point, based on improvement in the non-caeruloplasmin-bound copper, with mean reduction $72 \%$ from baseline $(-2.4 \mu \mathrm{mol} / \mathrm{L}, \mathrm{P}<0.001) .{ }^{51}$ Mean UWDRS neurological subscores improved from 22.8 to $16.6(\mathrm{P}<0.001)$ but increased by at least five point in two patients. A phase III trial comparing Bis-choline Tetrathiomolybdate to standard care (NCT03403205) is ongoing. 
Dimercaptosuccinic acid (DMSA) is a chelating agent widely used in China. A recent retrospective study comparing DMSA, in combination with Zinc, to Penicillamine in 158 patients with neurological presentations showed the proportion of patients with neurological improvements was higher ( $88 \%$ vs $59 \%$ ) and neurological worsening was lower (9\% vs $26 \%$ ) in the DMSA group. ${ }^{52}$ There are also reports of successfully using the parent compound, Dimercaprol (British Anti-Lewisite, BAL), as a rescue therapy for severe neurological worsening. ${ }^{53}$ It is lipid soluble and therefore needs to be administered intramuscularly but, unlike other chelating agents, can cross the blood-brain barrier.

\section{Paradoxical neurological worsening}

This unusual phenomenon is a major concern when initiating treatment. In a retrospective study that included 70 patients with neurological presentations, Litwin et al reported that $23 \%$ developed early neurological worsening with a mean delay of $2.3 \pm 1.9$ months. ${ }^{54}$ The deterioration was reversible in half of cases and higher baseline neurological severity (UWDRS 38.4 vs 22.3, P<0.01), more thalamic or brainstem lesions on MRI $(73 \%$ vs $33 \%, \mathrm{P}<0.01)$ and concurrent use of antipsychotic medication $(46 \%$ vs $5 \%, \mathrm{P}<0.01)$ were more common in patients who deteriorated. Brewer et al initially proposed the widely cited theory that paradoxical worsening is caused by transient elevation in blood and brain copper levels secondary to mobilisation of hepatic copper. Supporting evidence for this is however limited. Hartard et al found that CSF and non-caeruloplasmin-bound copper levels continued to decrease in a series of patients with paradoxical worsening. ${ }^{55}$ An alternative hypothesis that mobilisation of copper from a harmless, sequestered form (incorporated into metallothioneins) to other cellular compartments where it can cause oxidative damage has also been proposed.

The management of this challenging clinical scenario varies between clinicians and decisions on whether to increase or decrease doses, switch treatment or add another agent are usually guided by personal experience. Liver transplantation, usually reserved for patients with acute liver failure of decompensated liver disease, was recently evaluated in 18 patients with neurological worsening on chelation therapy. ${ }^{56}$ These patients had severe neurological disability, with a median modified Rankin scale (mRS) score 5 and median UWDRS score 105, at baseline. At last follow up, these had decreased to median mRS score 1.5 and median UWDRS score $36(\mathrm{P}<0.001)$. Eight patients had major improvement, four patients had moderate improvement, two were stable and four died. The mechanism by which liver transplantation can ameliorate neurological involvement is currently unclear, as is the role for this treatment in clinical practice.

\section{Experimental therapies}


Novel copper chelators, including DPM-1001, a protein-tyrosine phosphatase inhibitor with high copper affinity, Chel2, a hepatocyte-directed pro-chelator that releases a tristhiolate chelator intracellularly, and $\alpha$-lipoic acid, an endogenous sulphur-containing compound derived from caprylic acid, have been tested in animal or cell-based models of Wilson's disease with promising results. Methanobactin, a bacterial peptide with an exceptionally high affinity for copper, has been shown to prevent liver failure in $A T P 7 B$-deficient rats. ${ }^{57}$ An alternative strategy where a chelating agent, 8 hydroxyquinolone, is covalently bound to an indigestible biopolymer has also been shown to prevent intestinal absorption of copper in rodents. ${ }^{58}$

Targeted molecular therapies that restore the localisation and/or function of ATP7B are also being developed. A 20-residue peptide derived from $\alpha$ B-crystallin, a heat shock protein, has been shown to restore localisation of ATP7B to the TGN in cells transfected with ATP7B-H1069Q. ${ }^{59}$ Concilli et al recently interrogated the interactome of ATP7B and identified that the H1069Q mutation increases interactions with HSP70 thereby accelerating ATP7B degradation. Using a bioinformatic screen for drugs approved by the Food and Drug Administration, they found that Domperidone inhibits the function of HSP70 and improved localisation of ATP7B to the TGN and tolerance to exogenous copper in transfected cells. ${ }^{60}$ Whether this approach translates into a clinically-meaningful restoration of ATP7B function remains to be seen.

Finally, gene therapy using an adeno-associated viral (AAV) vector has shown encouraging results in animal models of Wilson's disease. Murillo et al transduced the liver of $A T P 7 B$ knockout mice with AAV8 encoding $A T P 7 B$ cDNA under the control of a liver specific promoter. They found a dose-dependent restoration of biliary copper excretion with normalisation of urinary copper output, hepatic copper content and liver function. ${ }^{61}$ Vectors containing a truncated form of the gene have shown similar results and a subsequent study has confirmed that AAV8-based therapy reduces cerebral copper content in knockout mice. ${ }^{62}$ An open-label phase I/II trial is due to start this year (NCT04537377). 


\section{FUTURE DIRECTIONS}

Significant progress has been made with our understanding of the genetics and cell biology of ATP7B dysfunction however the fundamental question of why only some patients primarily manifest with neurological disease remains. Novel approaches to exploring the genetic, epigenetic and wider metabolic influences that determine the initial presentation may be required. The lack of convincing neurological phenotype in animal models has also hindered our ability to characterise the pathogenesis of neurodegeneration and paradoxical neurological worsening in Wilson's disease. The use of induced pluripotent stem cells to study copper toxicity and ATP7B dysfunction in neurons and glia may provide novel insights here.

Delayed diagnoses remain an issue and confirming the sensitivity and specificity of novel diagnostic tests, including their potential use in newborn screening programmes, is a priority. Strategies to prevent and manage paradoxical worsening using novel treatments, existing chelating agents and liver transplantation also need to be clarified. We anticipate that imaging and wet biomarkers for neurological involvement will be increasingly used to guide chelation therapy. In an age of targeted molecular and gene therapies, we expect these will also play an important role in measuring neurological outcomes for these treatments in clinical trials.

\section{Contributors}

SS wrote the first draft of the manuscript. AP, OB, AC and TTW revised the manuscript.

\section{Funding}

SS is funded by the Guarantors of Brain, Association of British Neurologists and Reta Lila Weston Institute (no award/grant numbers).

\section{Competing interests}

None declared.

\section{Ethical approval}

Not required. 


\section{REFERENCES}

1. Lo C, Bandmann O. Epidemiology and introduction to the clinical presentation of Wilson disease. Handb Clin Neurol 2017;142:7-17.

2. Walshe JM, Yealland M. Chelation treatment of neurological Wilson's disease. $Q J \mathrm{Med}$ 1993;86(3):197-204.

3. Merle U, Schaefer M, Ferenci P, et al. Clinical presentation, diagnosis and long-term outcome of Wilson's disease: a cohort study. Gut 2007;56(1):115-20.

4. Telianidis J, Hung YH, Materia S, et al. Role of the P-Type ATPases, ATP7A and ATP7B in brain copper homeostasis. Front Aging Neurosci 2013;5:44.

5. Davies KM, Hare DJ, Cottam V, et al. Localization of copper and copper transporters in the human brain. Metallomics 2013;5(1):43-51.

6. Saito T, Okabe M, Hosokawa T, et al. Immunohistochemical determination of the Wilson Copper-transporting P-type ATPase in the brain tissues of the rat. Neurosci Lett 1999;266(1):13-6.

7. Poujois A, Mikol J, Woimant F. Wilson disease: brain pathology. Handb Clin Neurol 2017;142:77-89.

8. Petrukhin K, Lutsenko S, Chernov I, et al. Characterization of the Wilson disease gene encoding a P-type copper transporting ATPase: genomic organization, alternative splicing, and structure/function predictions. Hum Mol Genet 1994;3(9):1647-56.

9. Wallace DF, Dooley JS. ATP7B variant penetrance explains differences between genetic and clinical prevalence estimates for Wilson disease. Hum Genet 2020;139(8):1065-1075.

10. Kluska A, Kulecka M, Litwin T, et al. Whole-exome sequencing identifies novel pathogenic variants across the ATP7B gene and some modifiers of Wilson's disease phenotype. Liver Int 2019;39(1):177-186.

11. Coffey AJ, Durkie M, Hague S, et al. A genetic study of Wilson's disease in the United Kingdom. Brain 2013;136(5):1476-87.

12. Ferenci P, Stremmel W, Członkowska A, et al. Age and Sex but Not ATP7B Genotype Effectively Influence the Clinical Phenotype of Wilson Disease. Hepatology 2019;69(4):14641476.

13. Cheng $\mathrm{N}$, Wang $\mathrm{H}, \mathrm{Wu} \mathrm{W}$, et al. Spectrum of ATP7B mutations and genotype-phenotype correlation in large-scale Chinese patients with Wilson Disease. Clin Genet 2017;92(1):69-79. 
14. Schiefermeier M, Kollegger H, Madl C, et al. The impact of apolipoprotein E genotypes on age at onset of symptoms and phenotypic expression in Wilson's disease. Brain 2000;123(3):585-90.

15. Roy S, Ganguly K, Pal P, et al. Influence of Apolipoprotein E polymorphism on susceptibility of Wilson disease. Ann Hum Genet 2018;82(2):53-59.

16. Parisi S, Polishchuk EV, Allocca S, et al. Characterization of the most frequent ATP7B mutation causing Wilson disease in hepatocytes from patient induced pluripotent stem cells. Sci Rep 2018;8(1):6247.

17. Huster D, Kühne A, Bhattacharjee A, et al. Diverse functional properties of Wilson disease ATP7B variants. Gastroenterology 2012;142(4):947-956.e5.

18. Roy S, McCann CJ, Ralle M, et al. Analysis of Wilson disease mutations revealed that interactions between different ATP7B mutants modify their properties. Sci Rep 2020;10(1):13487.

19. Litwin T, Gromadzka G, Szpak GM, et al. Brain metal accumulation in Wilson's disease. $J$ Neurol Sci 2013;329(1-2):55-8.

20. Przybyłkowski A, Gromadzka G, Chabik G, et al. Liver cirrhosis in patients newly diagnosed with neurological phenotype of Wilson's disease. Funct Neurol 2014;29(1):23-9.

21. Ferenci P, Steindl-Munda P, Vogel W, et al. Diagnostic value of quantitative hepatic copper determination in patients with Wilson's Disease. Clin Gastroenterol Hepatol 2005;3(8):811-8.

22. Czlonkowska A, Tarnacka B, Litwin T, et al. Wilson's disease-cause of mortality in 164 patients during 1992-2003 observation period. J Neurol 2005;252(6):698-703.

23. Reed E, Lutsenko S, Bandmann O. Animal models of Wilson disease. J Neurochem 2018;

24. Zischka H, Lichtmannegger J, Schmitt S, et al. Liver mitochondrial membrane crosslinking and destruction in a rat model of Wilson disease. J Clin Invest 2011;121(4):1508-18.

25. Borchard S, Bork F, Rieder T, et al. The exceptional sensitivity of brain mitochondria to copper. Toxicol In Vitro 2018;51:11-22.

26. Medici V, Sarode GV, Napoli E, et al. mtDNA depletion-like syndrome in Wilson disease. Liver Int 2020;40(11):2776-2787.

27. Dezortova M, Lescinskij A, Dusek P, et al. Multiparametric Quantitative Brain MRI in Neurological and Hepatic Forms of Wilson's Disease. J Magn Reson Imaging 2020;51(6):1829-1835.

28. Dusek P, Bahn E, Litwin T, et al. Brain iron accumulation in Wilson disease: a post mortem 7 Tesla MRI - histopathological study. Neuropathol Appl Neurobiol 2017;43(6):514-532. 
29. Fu H, Hardy J, Duff KE. Selective vulnerability in neurodegenerative diseases. Nat Neurosci 2018;21(10):1350-1358.

30. McGee TP, Houston CM, Brickley SG. Copper block of extrasynaptic GABAA receptors in the mature cerebellum and striatum. J Neurosci 2013;33(33):13431-5.

31. O'Brien M, Reilly M, Sweeney B, et al. Epidemiology of Wilson's disease in Ireland. Mov Disord 2014;29(12):1567-8.

32. Millard H, Zimbrean P, Martin A. Delay in Diagnosis of Wilson Disease in Children With Insidious Psychiatric Symptoms: A Case Report and Review of the Literature. Psychosomatics 2015;56(6):700-5.

33. Ferenci P, Caca K, Loudianos G, et al. Diagnosis and phenotypic classification of Wilson disease. Liver Int 2003;23(3):139-42.

34. Steindl P, Ferenci P, Dienes HP, et al. Wilson's disease in patients presenting with liver disease: a diagnostic challenge. Gastroenterology 1997;113(1):212-8.

35. Espinos C, Ferenci P. Are the new genetic tools for diagnosis of Wilson disease helpful in clinical practice? JHEP Rep 2020;2(4):100114.

36. Członkowska A, Rodo M, Wierzchowska-Ciok A, et al. Accuracy of the radioactive copper incorporation test in the diagnosis of Wilson disease. Liver Int 2018;38(10):1860-1866.

37. Lyon TD, Fell GS, Gaffney D, et al. Use of a stable copper isotope $(65 \mathrm{Cu})$ in the differential diagnosis of Wilson's disease. Clin Sci (Lond) 1995;88(6):727-32.

38. Poujois A, Trocello JM, Djebrani-Oussedik N, et al. Exchangeable copper: a reflection of the neurological severity in Wilson's disease. Eur J Neurol 2017;24(1):154-160.

39. Woimant F, Djebrani-Oussedik N, Poujois A. New tools for Wilson's disease diagnosis: exchangeable copper fraction. Ann Transl Med 2019;7(Suppl 2):S70.

40. Collins CJ, Yi F, Dayuha R, et al. Direct Measurement of ATP7B Peptides is Highly Effective in the Diagnosis of Wilson Disease. Gastroenterology 2021;

41. Hahn SH. Population screening for Wilson's disease. Ann N Y Acad Sci 2014;1315:64-9.

42. Czlonkowska A, Litwin T, Dziezyc K, et al. Characteristics of a newly diagnosed Polish cohort of patients with neurological manifestations of Wilson disease evaluated with the Unified Wilson's Disease Rating Scale. BMC Neurol 2018;18(1):34.

43. Litwin T, Gromadzka G, Członkowska A, et al. The effect of gender on brain MRI pathology in Wilson's disease. Metab Brain Dis 2013;28(1):69-75.

44. Dusek P, Smolinski L, Redzia-Ogrodnik B, et al. Semiquantitative Scale for Assessing Brain MRI Abnormalities in Wilson Disease: A Validation Study. Mov Disord 2020;35(6):994-1001. 
45. Shribman S, Heller C, Burrows M, et al. Plasma Neurofilament Light as a Biomarker of Neurological Involvement in Wilson's Disease. Mov Disord 2021;36(2):503-508.

46. Lin J, Zheng Y, Liu Y, et al. Higher Concentration of Plasma Glial Fibrillary Acidic Protein in Wilson Disease Patients With Neurological Manifestations. Mov Disord 2021;

47. Broniek-Kowalik K, Dzieżyc K, Litwin T, et al. Anterior segment optical coherence tomography (AS-OCT) as a new method of detecting copper deposits forming the KayserFleischer ring in patients with Wilson disease. Acta Ophthalmol 2019;97(5):e757-e760.

48. Langwinska-Wosko E, Litwin T, Dziezyc K, et al. Optical coherence tomography as a marker of neurodegeneration in patients with Wilson's disease. Acta Neurol Belg 2017;117(4):867871.

49. Appenzeller-Herzog C, Mathes T, Heeres MLS, et al. Comparative effectiveness of common therapies for Wilson disease: A systematic review and meta-analysis of controlled studies. Liver Int 2019;39(11):2136-2152.

50. Brewer GJ, Askari F, Lorincz MT, et al. Treatment of Wilson disease with ammonium tetrathiomolybdate: IV. Comparison of tetrathiomolybdate and trientine in a double-blind study of treatment of the neurologic presentation of Wilson disease. Arch Neurol 2006;63(4):521-7.

51. Weiss KH, Askari FK, Czlonkowska A, et al. Bis-choline tetrathiomolybdate in patients with Wilson's disease: an open-label, multicentre, phase 2 study. Lancet Gastroenterol Hepatol 2017;2(12):869-876.

52. Zhang J, Xiao L, Yang W. Combined sodium Dimercaptopropanesulfonate and zinc versus Dpenicillamine as first-line therapy for neurological Wilson's disease. BMC Neurol 2020;20(1):255.

53. Walshe JM, Munro NA. Zinc-induced deterioration in Wilson's disease aborted by treatment with penicillamine, dimercaprol, and a novel zero copper diet. Arch Neurol 1995;52(1):10-1.

54. Litwin T, Dzieżyc K, Karliński M, et al. Early neurological worsening in patients with Wilson's disease. J Neurol Sci 2015;355(1-2):162-7.

55. Hartard C, Weisner B, Dieu C, et al. Wilson's disease with cerebral manifestation: monitoring therapy by CSF copper concentration. J Neurol 1993;241(2):101-7.

56. Poujois A, Sobesky R, Meissner WG, et al. Liver transplantation as a rescue therapy for severe neurologic forms of Wilson disease. Neurology 2020;94(21):e2189-e2202.

57. Lichtmannegger J, Leitzinger C, Wimmer R, et al. Methanobactin reverses acute liver failure in a rat model of Wilson disease. J Clin Invest 2016;126(7):2721-35.

58. Vetrik M, Mattova J, Mackova H, et al. Biopolymer strategy for the treatment of Wilson's disease. J Control Release 2018;273:131-138. 
59. Allocca S, Ciano M, Ciardulli MC, et al. An $\alpha \mathrm{B}-$ Crystallin Peptide Rescues Compartmentalization and Trafficking Response to Cu Overload of ATP7B-H1069Q, the Most Frequent Cause of Wilson Disease in the Caucasian Population. Int J Mol Sci 2018;19(7)

60. Concilli M, Petruzzelli R, Parisi S, et al. Pharmacoproteomics pinpoints HSP70 interaction for correction of the most frequent Wilson disease-causing mutant of ATP7B. Proc Natl Acad Sci U S A 2020;117(51):32453-32463.

61. Murillo O, Luqui DM, Gazquez C, et al. Long-term metabolic correction of Wilson's disease in a murine model by gene therapy. J Hepatol 2016;64(2):419-426.

62. Uerlings R, Moreno D, Murillo O, et al. Brain copper storage after genetic long-term correction in a mouse model of Wilson disease. Neurol Genet 2018;4(3):e243. 


\section{FIGURE LEGEND}

Figure 1. Axial and coronal FLAIR images demonstrating signal abnormality, which appears heterogeneously hyperintense, in the basal ganglia, thalami and brainstem of a patient presenting with hypomania, tremor and ataxia (A and B) and in the basal ganglia of a patient presenting with dysarthria, dystonia and parkinsonism (C and D). FLAIR, fluid attenuated inversion recovery. 


\section{TABLES}

\section{Table 1. Clinical features in patients with neurological presentations}

\begin{tabular}{lll}
\hline Clinical feature & Frequency & Comments \\
\hline Neurological & & \\
Dysarthria & $52-91 \%$ & Often associated with drooling and/or dysphagia \\
Postural tremor & $55-72 \%$ & May be worse with arms flexed (wing-beating tremor) \\
Dystonia & $20-69 \%$ & Facial/oromandibular involvement common (risus sardonicus) \\
Parkinsonism & $45-58 \%$ & \\
Ataxia & $28-54 \%$ & Cerebellar eye signs may be seen \\
Chorea & $11-16 \%$ & \\
Seizures & $4-15 \%$ & Usually younger patients \\
Executive dysfunction & - & Often manifests as decline in performance at school or work \\
Psychiatric & & \\
Incongruous behaviour & $13-25 \%$ & \\
Irritability & $13-18 \%$ & May be misattributed to puberty in adolescent patients \\
Personality change & $8-26 \%$ & \\
Depression & $4-21 \%$ & \\
Elation/Hypomania & $5-13 \%$ & Sometimes misdiagnosed as bipolar affective disorder \\
Anxiety & $2-7 \%$ & \\
Delusions & $1-2 \%$ & \\
Hepatic & & \\
Transaminitis & $30 \%$ & Alanine transaminase (ALT) $>$ 40 IU/L \\
Thombocytopenia & $50 \%$ & Platelet count < 140 x $10^{9} / \mathrm{L}$ \\
Coagulopathy & $65 \%$ & International normalised ratio $>1.2$ \\
Splenomegaly & $30 \%$ & Identified on ultrasound \\
Oesophageal varices & $48 \%$ & \\
Ophthalmological & $85-90 \%$ & Confirmed on slit lamp examination \\
Kayser-Fleischer rings & $<5 \%$ & \\
Sunflower cataracts & & \\
\hline
\end{tabular}

Frequency refers to number of patients with a given clinical feature relative to patients with neurological presentations. References are provided in the supplementary material. 
Table 2. Quantitative MRI studies on Wilson's disease

\begin{tabular}{|c|c|c|c|}
\hline Study & Participants & Methods & Key findings \\
\hline \multicolumn{4}{|c|}{ Tl-weighted (structural) imaging } \\
\hline Stezin et al & $10 \mathrm{WD}, 11 \mathrm{HC}$ & Voxel-based morphometry & Volume reduced in striatum, pallidum, thalamus, cerebellum and some cortical areas \\
\hline Smolinski et $a l^{*}$ & $48 \mathrm{WD}$ & Tissue-type segmentation & $\begin{array}{l}\text { UWDRS negatively correlates with volume of deep GM, peripheral GM and WM } \\
\text { Non-caeruloplasmin-bound copper negatively correlates with total brain volume }\end{array}$ \\
\hline Zou et al & $27 \mathrm{WD}, 24 \mathrm{HC}$ & $\begin{array}{l}\text { Volumetric/ROI } \\
\text { Morphometric }\end{array}$ & $\begin{array}{l}\text { Volume reduced in caudate, putamen, pallidum, thalamus, RN and SN } \\
\text { Modified Young scale negatively correlates with volume of putamen and pallidum }\end{array}$ \\
\hline Song et al & $30 \mathrm{WD}, 25 \mathrm{HC}$ & $\begin{array}{l}\text { Volumetric/ROI } \\
\text { Cortical thickness }\end{array}$ & $\begin{array}{l}\text { Volume reduced in subcortical nuclei, diffuse WM and several other GM regions } \\
\text { Cortical thickness reduced in left precentral gyrus and left insula } \\
\text { Global Assessment Scale negatively correlates with volume of several ROI, including putamen }\end{array}$ \\
\hline Tinaz et al & $30 \mathrm{WD}, 30 \mathrm{HC}$ & $\begin{array}{l}\text { Volumetric/ROI } \\
\text { Cortical thickness }\end{array}$ & $\begin{array}{l}\text { Volume reduced in striatum and WM ; Volume of striatum also lower in nWD than hWD } \\
\text { Cortical thickness redcued in bilateral superior frontal gyrus }\end{array}$ \\
\hline Viveiros et al & $20 \mathrm{WD}, 20 \mathrm{HC}$ & Volumetric/ROI & $\begin{array}{l}\text { Volume reduced in basal ganglia, amygdala, hippocampus, cerebellum, WM, MCP, SCP } \\
\text { Volume of striatum lower in nWD than hWD }\end{array}$ \\
\hline Hu et al & $22 \mathrm{WD}, 26 \mathrm{HC}$ & Volumetric/ROI & Volume reduced in basal ganglia and occipital fusiform cortex and increased in visual association cortex \\
\hline Dusek et al & $29 \mathrm{WD}, 26 \mathrm{HC}$ & $\begin{array}{l}\text { Deformation and surface- } \\
\text { based morphometry }\end{array}$ & $\begin{array}{l}\text { Volume decreased in basal ganglia, brainstem, internal capsule, corticospinal tract, motor and visual cortex } \\
\text { UWDRS negatively correlates with volume of putamen, pallidum, RN and SN }\end{array}$ \\
\hline \multicolumn{4}{|c|}{ Susceptibility-weighted imaging } \\
\hline Fritzsch et al & $11 \mathrm{WD}, 10 \mathrm{HC}$ & QSM values/ROI & QSM values increased in basal ganglia, $\mathrm{RN}$ and $\mathrm{SN}$; also higher in $\mathrm{SN}$ and $\mathrm{RN}$ of hWD than controls \\
\hline Bai et al & $23 \mathrm{WD}, 23 \mathrm{HC}$ & Phase values/ROI & Phase values reduced in caudate, putamen, thalamus, $\mathrm{RN}$ and $\mathrm{SN}$ \\
\hline Zhou et al & $30 \mathrm{WD}, 20 \mathrm{HC}$ & Phase values/ROI & $\begin{array}{l}\text { Phase values reduced in caudate, pallidum and SN } \\
\text { Tremor score negatively correlated with phase value in caudate }\end{array}$ \\
\hline Yang et al & $33 \mathrm{WD}, 18 \mathrm{HC}$ & Phase values/ROI & Phase values reduced in caudate, putamen, pallidum, thalamus, $\mathrm{RN}$ and $\mathrm{SN}$ \\
\hline $\begin{array}{l}\text { Dezortova et al } \\
\text { Dusek et al }\end{array}$ & $\begin{array}{l}38 \mathrm{WD}, 26 \mathrm{HC} \\
29 \mathrm{WD}, 26 \mathrm{HC}\end{array}$ & $\begin{array}{l}\text { QSM values/ROI } \\
\text { QSM values/ROI }\end{array}$ & $\begin{array}{l}\text { QSM values increased in caudate, putamen, pallidum and thalamus in nWD compared to hWD and controls } \\
\text { UWDRS did not correlate with mean QSM values in deep GM nuclei }\end{array}$ \\
\hline \multicolumn{4}{|c|}{ Diffusion-weighted imaging } \\
\hline Jadav et al & $15 \mathrm{WD}, 15 \mathrm{HC}$ & DTI/ROI & $\begin{array}{l}\text { MD increased and FA decreased in WM; } \\
\text { Neurological severity correlates with MD in internal capsule and frontal and occipital WM }\end{array}$ \\
\hline Lawrence et $a l^{* \dagger}$ & $35 \mathrm{WD}$ & DTI/TBSS & Decreases in FA and increases in $\mathrm{MD}, \mathrm{AD}$ and $\mathrm{RD}$ improve over first two years of treatment \\
\hline Zhou et al & $70 \mathrm{WD}, 20 \mathrm{HC}$ & DTI/ROI & FA and fibre volumes lower in nWD than hWD \\
\hline Wang et al & $35 \mathrm{WD}, 30 \mathrm{HC}$ & DTI/ROI & FA increased in subcortical GM nuclei and decreased in thalamus and WM \\
\hline Song et al & $24 \mathrm{WD}, 25 \mathrm{HC}$ & NODDI/ROI & $\begin{array}{l}\text { Vic and OBI reduced and Viso increased in basal ganglia and thalamus } \\
\text { Clinical severity negatively correlates with Vic in putamen and ODI in pallidum }\end{array}$ \\
\hline Dong et al & $30 \mathrm{WD}, 35 \mathrm{HC}$ & DTI/ROI & $\begin{array}{l}\text { FA increased in subcortical GM nuclei and decreased in right cerebellum and left middle frontal gyrus } \\
\text { Event-based prospective memory and digit span correlate with FA in left thalamus and putamen }\end{array}$ \\
\hline Hu et al & $22 \mathrm{WD}, 26 \mathrm{HC}$ & DTI/ROI (tract-specific) & $\begin{array}{l}\text { Increased } \mathrm{MD}, \mathrm{RD} \text { and } \mathrm{AD} \text { and decreased } \mathrm{FA} \text { in various association/limbic tracts } \\
\text { Associations between } \mathrm{AD} \text { and } \mathrm{FA} \text { and event-based and time-based prospective memory tasks in some tracts }\end{array}$ \\
\hline
\end{tabular}

Studies are listed in chronological order under each subheading. References are provided in the supplementary material.

* indicates study included newly-diagnosed patients.

${ }^{\dagger}$ indicates study was longitudinal. 


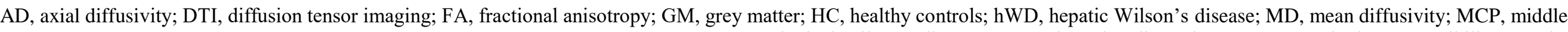

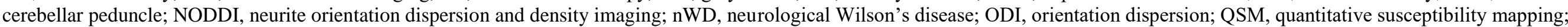

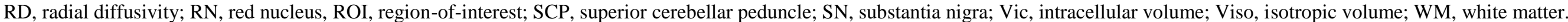


Table 3. MRS, SPECT and TCS studies on Wilson's disease

\begin{tabular}{|c|c|c|}
\hline Study & Participants & Key findings \\
\hline \multicolumn{3}{|c|}{ Magnetic resonance spectroscopy } \\
\hline Van Den Heuvel & $22 \mathrm{WD}, 13 \mathrm{HC}$ & $\begin{array}{l}\text { Decreases in } \mathrm{NAA} / \mathrm{Cr} \text { and } \mathrm{Cho} / \mathrm{CR} \text { in pallidum } \\
\text { Decreases in } \mathrm{mI} / \mathrm{Cr} \text { in pallidum of patients with porto-systemic shunting }\end{array}$ \\
\hline Kraft et al & $13 \mathrm{WD}, 12 \mathrm{HC}$ & No differences in putamen, GM or WM \\
\hline Lucato et al & $36 \mathrm{WD}, 37 \mathrm{HC}$ & $\begin{array}{l}\text { Decreases in } \mathrm{NAA} / \mathrm{Cr} \text { in basal ganglia, WM and } \mathrm{GM} \\
\text { Increases } \mathrm{mI} / \mathrm{Cr} \text { in basal ganglia }\end{array}$ \\
\hline Tarnacka et $a l^{*}$ & $37 \mathrm{WD}$ & $\begin{array}{l}\text { Increases in } \mathrm{Cho} / \mathrm{Cr} \text { and } \mathrm{Lip} / \mathrm{Cr} \text { and decreases in NAA/Cr in pallidum } \\
\text { Increases in } \mathrm{Cho} / \mathrm{Cr} \text { and } \mathrm{Lip} / \mathrm{Cr} \text { in thalamus (nWD vs } \mathrm{HC} \text { ) }\end{array}$ \\
\hline Tarnacka et al ${ }^{* \dagger}$ & $17 \mathrm{WD}$ & $\begin{array}{l}\mathrm{NAA} / \mathrm{Cr} \text { in pallidum increases with neurological recovery and decreases with deterioration } \\
\mathrm{mI} / \mathrm{Cr} \text { and } \mathrm{Glx} / \mathrm{Cr} \text { in pallidum decrease with liver failure }\end{array}$ \\
\hline Tarnacka et al & $12 \mathrm{HZ}, 21 \mathrm{HC}$ & Increases in $\mathrm{Glx} / \mathrm{Cr}$ and $\mathrm{Lip} / \mathrm{Cr}$ in pallidum and thalamus of heterozygous carriers \\
\hline Pulai et al & $38 \mathrm{WD}, 32 \mathrm{HC}$ & Decreases in $\mathrm{NAA} / \mathrm{Cr}$ and $\mathrm{Cho} / \mathrm{Cr}$ in basal ganglia \\
\hline Alkhalik Basha et $a l^{*}$ & $26 \mathrm{WD}, 26 \mathrm{HC}$ & Decreases in NAA, Cho, Cr, NAA/Cho, NAA/Cr, $\mathrm{Cho} / \mathrm{Cr}$ in pallidum \\
\hline \multicolumn{3}{|c|}{ Single photon emission computed tomography } \\
\hline Barthel et al & $46 \mathrm{WD}, 10 \mathrm{HC}$ & Neurological severity correlates with ${ }^{123}$ I- $\beta$-CIT and ${ }^{123}$ I-IBZM binding in striatum \\
\hline Hermann et al & $37 \mathrm{WD}$ & Fine motor ability correlates with ${ }^{123} \mathrm{I}-\beta$-CIT binding in putamen \\
\hline Eggers et al & $23 \mathrm{WD}$ & Hamilton rating scale for depression correlates with ${ }^{123}$ I- $\beta$-CIT binding in thalamus-hypothalamus \\
\hline Wang et al & $31 \mathrm{WD}, 12 \mathrm{HC}$ & Decreases in ${ }^{99 \mathrm{~m}} \mathrm{Tc}-\mathrm{TRODAT}-1$ binding in posterior putamina \\
\hline Piga et al ${ }^{*}$ & $25 \mathrm{WD}, 24 \mathrm{HC}$ & Decreases in ${ }^{99 \mathrm{~m}} \mathrm{Tc}-\mathrm{ECD}$ uptake in the caudate, putamen and scattered cortical areas \\
\hline Eo et al & $9 \mathrm{WD}, 17 \mathrm{HC}$ & Decreases in ${ }^{123}$ I- $\beta$-CIT binding in striatum \\
\hline \multicolumn{3}{|l|}{ Transcranial sonography } \\
\hline Walter et al & $21 \mathrm{WD}$ & $\begin{array}{l}\text { HE in putamen seen in all } 18 \mathrm{nWD} \text { cases } \\
\text { HE in putamen and thalamus and third ventricular width correlate with disease severity }\end{array}$ \\
\hline Svetel at al & $54 \mathrm{WD}$ & HE in SN and third ventricular width correlate with disease severity \\
\hline Maskova et al & $22 \mathrm{WD}, \mathrm{HC}, 16 \mathrm{EOPD}$ & $\begin{array}{l}\text { HE higher in putamen in WD than HC and EOPD } \\
\text { HE higher in SN in EOPD than WD and HC }\end{array}$ \\
\hline Tribl et al & $40 \mathrm{WD}, 49 \mathrm{HC}$ & $\begin{array}{l}\text { HE in putamen differentiates WD and HC with area-under-curve } 0.95 \\
\text { HE in putamen correlates with dystonia and dysarthria scores } \\
\text { Third ventricular width correlates with UWDRS, ACE-R, MMSE scores }\end{array}$ \\
\hline Skowronska et $a l^{* \dagger}$ & $41 \mathrm{WD}$ & $\mathrm{HE}$ in SN but not putamen improves with treatment \\
\hline Skowronska et al & $34 \mathrm{HZ}, 18 \mathrm{HC}$ & HE in putamen seen in $75 \%$ of heterozygous carriers \\
\hline Skoloudik et al & $22 \mathrm{WD}, 24 \mathrm{HC}$ & HE in insula in WD and correlates with HE in putamen \\
\hline
\end{tabular}

Studies are listed in chronological order under each subheading. References are provided in the supplementary material.

* indicates study included newly-diagnosed patients.

${ }^{\dagger}$ indicates study was longitudinal. 


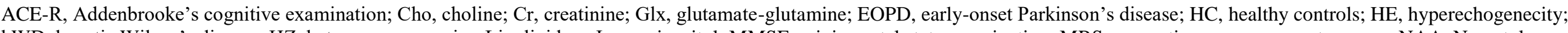

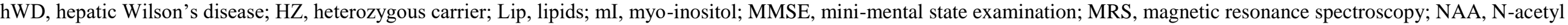

aspartate; nWD, neurological Wilson's disease; SN, substantia nigra, SPECT, single photon emission computed tomography; TCS, transcranial sonography. 